\title{
Docking to Pallets with \\ Feedback from a Sheet-of-Light Range Camera J. Nygårds ${ }^{1}$, T. Högström ${ }^{1,2}$ and Å. Wernersson ${ }^{3}$
}

\author{
${ }^{1}$ Robotics, Linköping University, \\ SE-58183 LINKOPING, SWEDEN \\ email:jonny@ikp.liu.se, URL: http://idefix.ikp.liu.se/rames/ \\ ${ }^{2}$ Scandicraft Systems \\ Teknikringen 6 \\ S-583 30 Linköping, SWEDEN \\ ${ }^{3}$ Robotics, Luleå Univ of Technology, \\ SE-97187 LULEA, SWEDEN \\ email:akewe@sm.luth.se, URL: http://www.sm.luth.se/csee/ra/
}

\section{Abstract}

The problem studied is feedback for docking a mobile robot, or AGV, to a pallet. The pallet is of known size but with an essentially unknown load. The pallet has an initial uncertainty in pose (position and orientation) of the order $\pm 15 \mathrm{~cm}$ and \pm 20 degrees. The docking error is required to be within $\pm 1 \mathrm{~cm}$ and \pm 1 degree with "very low" failure rate. For the docking a combination of a range camera and a video camera is used. In this paper the range cameras is emphasized.

Experimental results from this work in progress are presented. Successful docking has been made with typical $\pm 5 \mathrm{~mm}$ as errors. Currently one weak part is the integration with the control system on board the robot. Our persistent experience from this and earlier tests is that the weak part when using non-contact sensing for feedback in robots is the association problem. It should be mentioned that the resolution of a range camera is strongly distance dependent. One finding in the paper is that this type of docking is feasible and can be made selfmonitoring. The feedback laws are to be improved.

Keywords:Mobile robot, pallet, docking, range-camera, laser-vision, Radon transform, Sensor Fusion, Kalman filtering, Feedback, Association errors, Robustness

\section{Introduction}

The task is automation of pallet docking for insertion into an AGV factory automation system. The initial uncertainty in the pallet position is specified to be $\pm 15 \mathrm{~cm}$ and \pm 20 degrees, while the docking error is required to be within $\pm 1 \mathrm{~cm}$ and \pm 1 degree with "very low" failure rate. This paper is on the signal/image processing and control used to achieve those goals.

During an approach to the pallet the range camera is used from approximately $4 \mathrm{~m}$ down to $1 \mathrm{~m}$. In this interval the range resolution varies within a factor of 15 . Thus the uncertainty volume in the $(x, y, \theta)$-space varies by a factor of 1000 during the approach. A consequence is that the feedback law must be strongly range ('time') dependent. Traditional feedback design is not applicable.

The docking problem as such is well defined, but observe that it is the pose of the robot in the coordinates as defined by the pallet. Since the pose of the pallet is 'unknown' the robot does not know the orientation until it is very close to the pallet. Since the motion of the robot is non-holonomic, errors in cross range position are difficult to reduce.

\section{Equations of Motion and Uncertainty Propagation}

The docking problem is well defined in the coordinates of the pallet. Thus, we start by the equations for how the uncertainties are transformed and propagates.

Consider the relative posture $X_{k}=\left[\begin{array}{lll}x_{k} & y_{k} & \theta_{k}\end{array}\right]^{T}$ expressing the posture of a stationary object relative to the moving platform in Figure 1. The desired translation $T_{\mathrm{k}}$ and rotation $\Omega_{k}$ of the platform are expressed in the coordinate system of time $\mathrm{k}$ and combined into the control signal $u_{k}$

The new relative posture after motion $X_{k+1}=\left[\begin{array}{lll}\xi_{k+1} & \eta_{k+1} & \theta_{k+1}\end{array}\right]^{T}$ is then given by the motion or coordinate transform equation:

$$
\begin{aligned}
& X_{k+1}=f\left(X_{k}, u_{k}\right)+w_{k}=R\left(-\Omega_{k}\right)\left(X_{k}-u_{k}\right)+w_{k} \\
& \text { where } \quad u_{k}=\left[\begin{array}{lll}
T_{x k} & T_{y k} & \Omega_{k}
\end{array}\right] \\
& R\left(-\Omega_{k}\right)=\left[\begin{array}{ccc}
\cos \left(\Omega_{k}\right) & \sin \left(\Omega_{k}\right) & 0 \\
-\sin \left(\Omega_{k}\right) & \cos \left(\Omega_{k}\right) & 0 \\
0 & 0 & 1
\end{array}\right]
\end{aligned}
$$




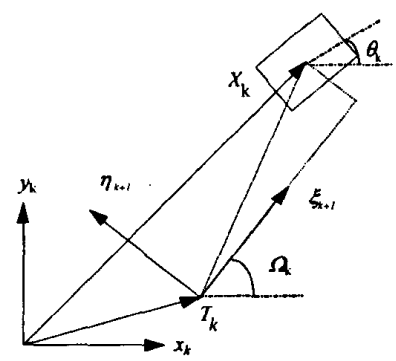

Figure 1 The relative co-ordinates for a robot moving towards a rectangular object at the upper right.

The change of variables in the position co-ordinates $\left(x_{k}, y_{k}\right)$ to the new co-ordinates $\left(\xi_{k+1}, \eta_{k+1}\right)$ is used to emphasise that the position is expressed in a new coordinate system.

A first order Taylor expansion of the prediction error about an estimated state $\hat{X}_{k \mid k}$ and desired control signal $\hat{u}_{k}$ gives:

$$
\begin{aligned}
\tilde{X}_{k+1 \mid k}= & X_{k+1}-\hat{X}_{k+1 \mid k} \approx f\left(\hat{X}_{k \mid k}, \hat{u}_{k}\right)+ \\
& F\left(\hat{X}_{k \mid k}, \hat{u}_{k}\right)\left(X_{k}-\hat{X}_{k \mid k}\right)+ \\
& \quad G\left(\hat{X}_{k \mid k}, \hat{u}_{k}\right)\left(u_{k}-\hat{u}_{k}\right)+w_{k}-f\left(\hat{X}_{k \mid k}, \hat{u}_{k}\right)
\end{aligned}
$$

with the Jacobians:

$$
\begin{aligned}
& F\left(X_{k}, u_{k}\right)=\frac{\partial f\left(X_{k}, u_{k}\right)}{\partial X_{k}}=R\left(-\Omega_{k}\right) \\
& G\left(\hat{X}_{k}, \hat{u}_{k}\right)=-R\left(-\hat{\Omega}_{k}\right)-\left[\begin{array}{l}
00 \\
00 \mathrm{~m} \\
00
\end{array}\right]
\end{aligned}
$$

where $\mathbf{m}=S\left(\mathbf{e}_{z}\right) R\left(-\hat{\mathbf{\Omega}}_{k}\right)\left(\hat{X}_{k}-\hat{u}_{k}\right)=\left[\begin{array}{lll}-\hat{\eta}_{k+1} & \hat{\xi}_{k+1} & 0\end{array}\right]^{r}$

The matrix $S(\bullet)$ is closely related to the partial derivatives of a rotation matrix and corresponds to the vector cross product with the unit vector $\mathbf{e}_{2},([\mathrm{SpV} 89] \mathrm{pp}$ 50-53), i. e.:

$\mathbf{a} \times \mathbf{b}=S(\mathbf{a}) \mathbf{b} \Rightarrow S(\mathbf{a})=\left[\begin{array}{ccc}0 & -a_{3} & a_{2} \\ a_{3} & 0 & -a_{1} \\ -a_{2} & a_{1} & 0\end{array}\right] \Rightarrow$

$S\left(\mathbf{e}_{z}\right)=\left[\begin{array}{ccc}0 & -1 & 0 \\ 1 & 0 & 0 \\ 0 & 0 & 0\end{array}\right]$
The resulting first order approximation of the error covariance is then

$$
\begin{aligned}
& E\left[\widetilde{X}_{k+1 \mid k} \widetilde{X}_{k+1 \mid k}^{T}\right] \approx \\
& F\left(\hat{X}_{k \mid k}, \hat{u}_{k}\right) E\left[\tilde{X}_{k \mid k} \tilde{X}_{k \mid k}^{\tau}\right] F\left(\hat{X}_{k \mid k}, \hat{u}_{k}\right)^{\prime}+ \\
& G\left(\hat{X}_{k \mid k}, \hat{u}_{k}\right) E\left[\tilde{u}_{k} \tilde{u}_{k}\right] G\left(\hat{X}_{k \mid k}, \hat{u}_{k}\right)^{\top}+E\left[w_{k} w_{k}^{T}\right]+ \\
& F\left(\hat{X}_{k \mid k}, \hat{u}_{k}\right) E\left[\widetilde{X}_{k \mid k} \widetilde{u}_{k}^{T}\right] g\left(\hat{X}_{k \mid k}, \hat{u}_{k}\right)^{\top}+ \\
& G\left(\hat{x}_{k \mid k}, \hat{u}_{k}\right) E\left[\tilde{u}_{k} \tilde{X}_{k \mid k}^{T}\right] F\left(\hat{X}_{k \mid k}, \hat{u}_{k}\right)
\end{aligned}
$$

where $E\left[\tilde{X}_{k} \tilde{u}_{k}^{T}\right]=0, E\left[w_{k} w_{k}^{T}\right]=Q$

$E\left[\tilde{X}_{k} \tilde{X}_{k}^{T}\right]=\Sigma_{k}$ and $E\left[\tilde{u}_{k} \tilde{u}_{k}^{T}\right]=\left[\begin{array}{ccc}\sigma_{x}^{2} & 0 & 0 \\ 0 & \sigma_{y}^{2} & 0 \\ 0 & 0 & \sigma_{\theta}^{2}\end{array}\right]$

i.e.

$E\left[\tilde{X}_{k+1 \mid k} \widetilde{X}_{k+1\} k}^{T}\right] \approx R\left(-\hat{\Omega}_{k}\right) k_{k} R\left(-\hat{\Omega}_{k}\right)+$

$R\left(-\hat{\Omega}_{k}\right) E\left[\tilde{u}_{k} \tilde{u}_{k}^{T}\right]_{R}\left(-\hat{\Omega}_{k}\right)+$

$\mathbf{m} \sigma_{\theta}^{2} \mathbf{m}^{T}+\left[\begin{array}{l}00 \\ 00 \mathbf{m} \\ 00\end{array}\right] \sigma_{\theta}^{2}+\left[\begin{array}{l}00 \\ 00 \mathbf{m} \\ 00\end{array}\right]^{T} \sigma_{\theta}^{2}+Q$

\section{Measurement information}

Consider measurements on a standard pallet; the laser will illuminate each of the three blocks in the front.

Each block will give a contribution to the position estimate. First consider a single block represented using a local coordinate system.

$$
\mathbf{R}_{1}=E\left[\widetilde{z}_{1} \tilde{z}_{1}^{T}\right] \approx\left[\begin{array}{ccc}
\sigma_{x 1} & 0 & 0 \\
0 & \sigma_{y 1} & 0 \\
0 & 0 & \sigma_{\theta 1}
\end{array}\right]
$$

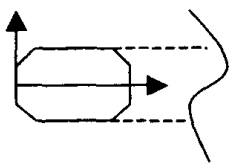

Figure 2 The position of a local coordinate system in each block. The coordinate system is attched at the middle of the front end of the block where the laser will illuminate the block. 

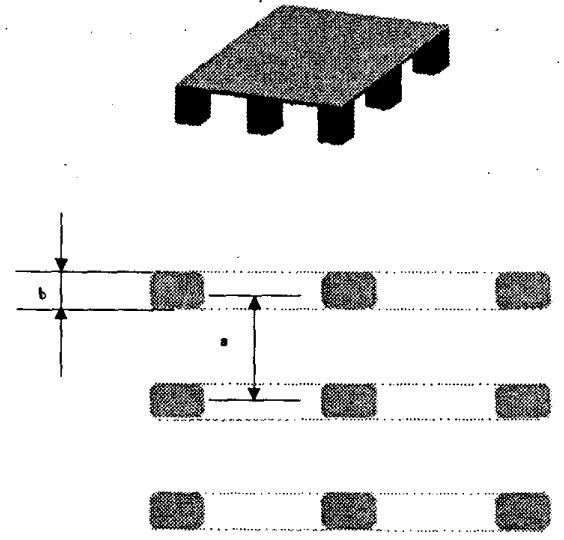

Figure 3 Standard pallet. The dimensions of the pallet follows the European Pool-pallet $8 \times 12$ standardi.e. the distance $\mathrm{a}$ is $0.35 \mathrm{~m}$ and $\mathrm{b}$ is $0.1 \mathrm{~m}$ the figure is slightly misleading since the middle block is larger having $a$ width of $0.145 \mathrm{~m}$.

The geometry of the range camera is defined by the parameters:

$h=$ Baseline distance i. e. the perpendicular distance from the laser plane to the pin-hole of the camera optics.

$\phi=$ Angle between the optical axis and the light plane.

$f=$ Focal distance between image plane and pinhole.

Assume that a detected point in the image plane is modeled as:

$p \in N\left(p-\bar{p}, \sigma_{p}^{2}\right)$

$q \in N\left(q-\bar{q}, \sigma_{q}^{2}\right)$

where the notation $N\left(x-\bar{x}, \sigma_{x}^{2}\right)$ denotes a Gaussian variable $x$ with mean $\bar{x}$ and variance $\sigma_{x}^{2}$. Then the uncertainties of range camera measurements of a single block is approximated as [NNW97], [NW98]:

$\sigma_{x}^{2} \approx \frac{x_{c}^{4}}{h^{2} f^{2}} \sigma_{p}^{2} \frac{x_{c}}{f b}$ $\sigma_{y}^{2} \approx \frac{x_{c}^{2}}{f^{2}} \sigma_{q}^{2}$

$\sigma_{\theta}^{2} \approx \frac{12 \sigma_{x}^{2}}{b^{2}}=\frac{12 x_{c}^{4}}{b^{2} h^{2} f^{2}} \sigma_{p}^{2} \frac{x_{c}}{f b}$

Here the number of measurements falling on the block is approximated as:

$n \approx \frac{f b}{x_{c}}$

The coordinate $x_{c}$ is the distance from the lens to the point projected on the optical axis i. e. the coordinate in a direction perpendicular to the image plane. i.e.

$x_{c}=x \cos (\varphi)+h \sin (\varphi)$

using eqn (6) the estimate is transformed to the pallet coordinate system with covariance according to eqn (6):

$E\left[\widetilde{X}_{1} \tilde{X}_{1}^{T}\right] \approx E\left[\tilde{u}_{1} \tilde{u}_{1}^{T}\right]+\left[\begin{array}{lll}w & 0 & 0\end{array}\right] \sigma_{\theta}^{2}\left[\begin{array}{lll}w & 0 & 0\end{array}\right]^{\top}$

$+\left[\begin{array}{lll}0 & 0 & w \\ 0 & 0 & 0 \\ 0 & 0 & 0\end{array}\right] \sigma_{\theta}^{2}+\left[\begin{array}{ccc}0 & 0 & 0 \\ 0 & 0 & 0 \\ w & 0 & 0\end{array}\right] \sigma_{\theta}^{2}=$

$E\left[\widetilde{u}_{1} \tilde{u}_{1}^{T}\right]+\left[\begin{array}{ccc}w^{2} \sigma_{\theta}^{2} & 0 & w \sigma_{\theta}^{2} \\ 0 & 0 & 0 \\ w \sigma_{\theta}^{2} & 0 & 0\end{array}\right]=$

$\left[\begin{array}{ccc}\sigma_{x 1}+w^{2} \sigma_{\theta 1}^{2} & 0 & w \sigma_{\theta 1}^{2} \\ 0 & \sigma_{y 1} & 0 \\ w \sigma_{\theta 1}^{2} & 0 & \sigma_{\theta 1}\end{array}\right]$

$E\left[\widetilde{X}_{2} \widetilde{X}_{2}^{T}\right] \approx\left[\begin{array}{ccc}\sigma_{x 2} & 0 & 0 \\ 0 & \sigma_{y 2} & 0 \\ 0 & 0 & \sigma_{\theta 2}\end{array}\right]$

$E\left[\tilde{X}_{3} \tilde{X}_{3}^{T}\right] \approx\left[\begin{array}{ccc}\sigma_{x 3}+a^{2} \sigma_{\theta 3}^{2} & 0 & -a \sigma_{\theta 3}^{2} \\ 0 & \sigma_{y 3} & 0 \\ -a \sigma_{\theta 3}^{2} & 0 & \sigma_{\theta 3}\end{array}\right]$

As a first approximation assume that the position of each block is estimated with the same covariance then the combined information is: 


$$
\begin{aligned}
& {\left[\begin{array}{ccc}
\frac{\sigma_{x}^{2}}{3} & 0 & 0 \\
0 & \frac{\sigma_{y}^{2}}{3} & 0 \\
0 & 0 & \frac{\sigma_{x}^{2} \sigma_{\theta}^{2}}{3 \sigma_{x}^{2}+2 a^{2} \sigma_{\theta}^{2}}
\end{array}\right]=\left[\begin{array}{ccc}
\frac{\sigma_{x}^{2}}{3} & 0 & 0 \\
0 & \frac{\sigma_{y}^{2}}{3} & 0 \\
0 & 0 & \left(\frac{3}{\sigma_{\theta}^{2}}+\frac{2 a^{2}}{\sigma_{x}^{2}}\right)^{-1}
\end{array}\right]} \\
& \geq \operatorname{diag}\left(\frac{\sigma_{x}^{2}}{3}, \frac{\sigma_{y}^{2}}{3}, \frac{\sigma_{x}^{2}}{2 a^{2}}\right)
\end{aligned}
$$

\section{Experiments on feasibility and self- monitoring}

Experiments using a simple control law verify the feasibility of the task.

\subsection{Color filtering}

The color filtering is very simple; the green and blue color planes are subtracted from an amplified red color plane. By doing this most of the distortion of red color in the surrounding area of the laser-line is reduced. The geometry and a typical image are illustrated in Figures 1 and 3. This filtering should be replaced by a more elaborate scheme in the final version. However it works remarkably well and the result is good enough to perform these initial experiments.

\subsection{Finding the pallet center, Illustrated summary of the algorithm}

A Kalman filter is used to track the pallet position [AM79]. However, since the measurements on a closer distance are so much better than earlier measurements not much is gained by the combination with earlier information. Instead the primary function of the Kalman filter is as an aid in associating with the correct measurements of the pallet [BSF88]. A brief description of the signal processing along with illustrative pictures follows:

1. A prediction of the pallet position defines a region of interest (ROI) in the image, Figure 4.

2. To detect the line specifying the orientation of the pallet the Radon-transform is used. A neighborhood around the line detected as the maxima in the Radon transform is analyzed. Along the line, the pixel in every column with the highest intensity $( \pm 6$ pixel from the line in every column), is used in a linear regression to get an improved estimate of the pallets orientation. For every pixel (pi,qi) with laser detected, the real distance (xi,yi) with covariance $\mathrm{Ri}$ is computed and used in the linear regression giving the orientation and the distance to the pallet.
3. Finding the intervals where the laser-intensity peaks, and their width, it is possible to determine which interval is most likely to match the middle block on the pallet.

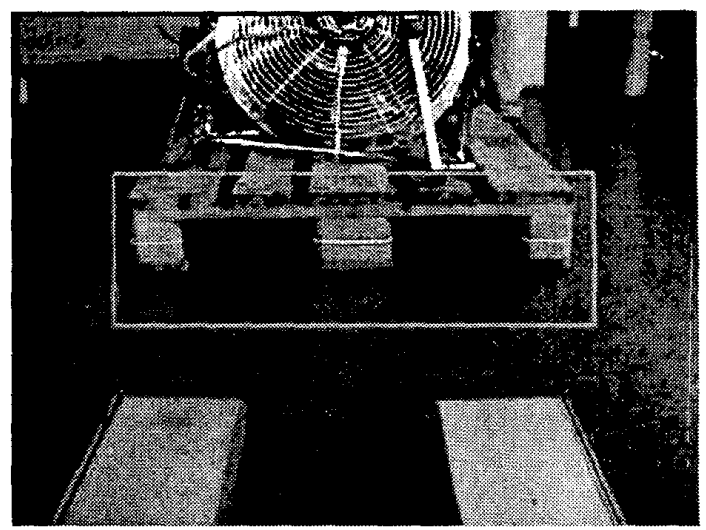

Figure 4 Using the prediction from previous images the laser line is searched for within a region of interest (ROI).

4. If there is a vertical edge, found by the EDGE operator, relatively close to the intensity-edges, this is assumed to be the best approximation for the edge of the block, Figure 5.

5. A projection to camera-coordinates is performed, and the midpoint and the distance to the block are computed. The results are in the coordinates of the robot, Figure 6.

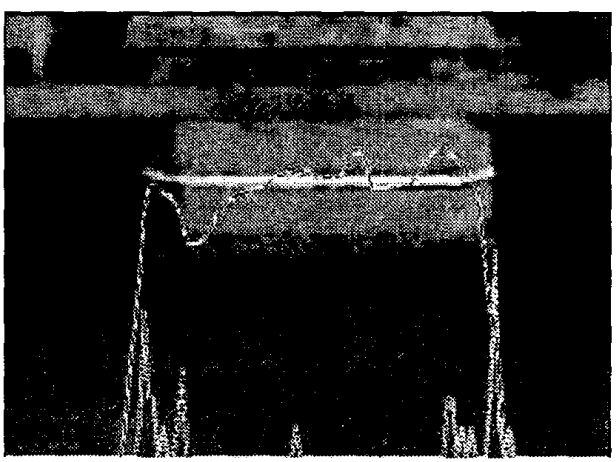

Figure 5 Within the interval where the laser is detected, edges are searched for in the intensity image. If suitable edges are detected they are used as features describing the width of the block in the middle. 


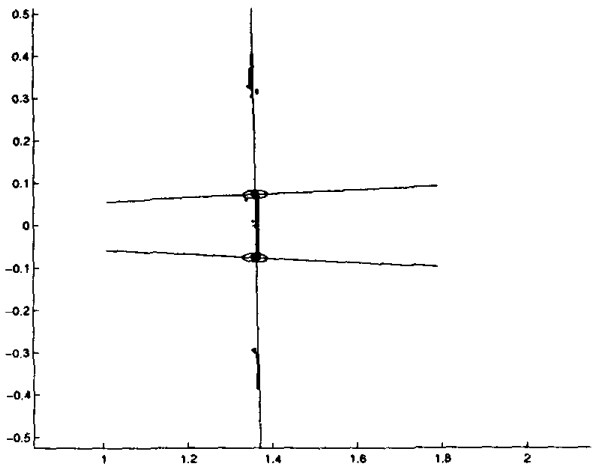

Figure 6 Projection to the range camera coordinates of the detected edges. (The cameralrobot is located at $y=0$ along the $y$-axis)

\section{Control law}

The current level of system integration only allows positioning commands to be sent to the robot with rather large steps (minimum in the order of $0.2 \mathrm{~m}$ ). The resulting control law then becomes a problem of choosing a few positions where new measurements and position corrections can be made. An important factor when choosing the position is the range varying uncertainty in orientation, Figure 7.

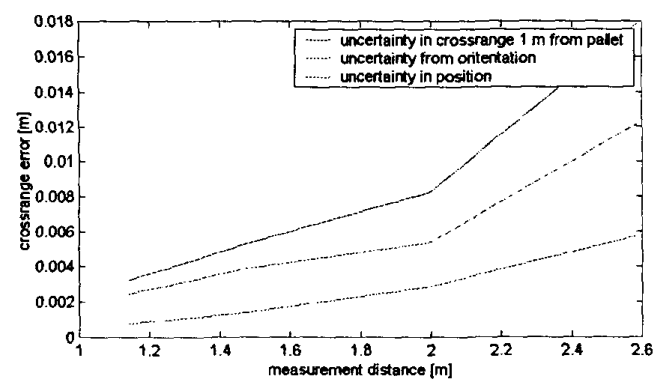

Figure 7 The cross-range uncertainty at the docking point as a function of the distance where the measurement is taken. The docking point is one meter in front of the pallet; at this point the lift must be able to proceed straight ahead or else back up. The uncertainty in orientation then results in an uncertainty in the crossrange position with the one-meter range acting as a lever.

The control law generates positions that are passed on to the robots internal control. There are a number of predefined distances from the pallet where new measurements are taken and a correction of the crossrange position is made. At each step the robot moves to the next distance with motion in the cross-range direction to the closest $3 \sigma$-limit if it is outside and towards the pallet if imnidatha limita nas Eimma

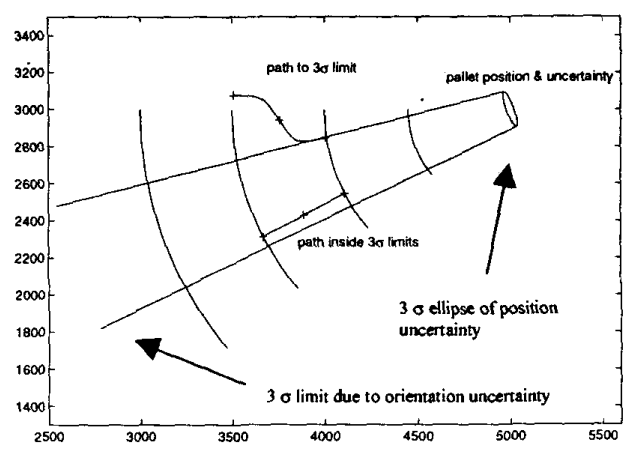

Figure 8 The figure shows the different path schemes for the robot depending on its current position relative to the pallet and the corresponding uncertainty. The uncertainties are exaggerated in the figure.

If the robot is too close to the pallet in order to steer up to any point, and the angle is too big to dock, the robot has to back up. New measurements are taken and a new attempt is made.

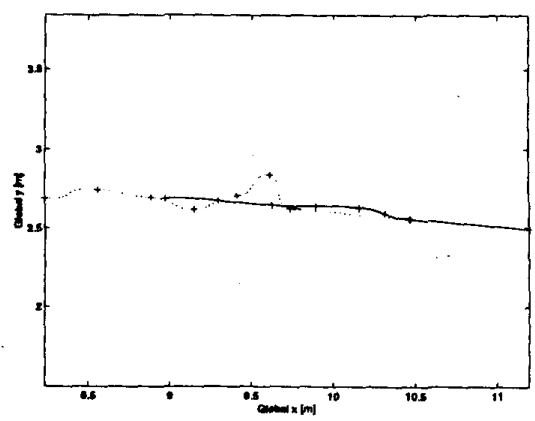

Figure 9 The trajectory traveled by the robot during the docking (according to the robot now. system). The dotted line represents the trajectory of the steering wheel while the solid line is the controlled reference point on the middle of the wheel coxis at the base of the forks.

In Figure 10 the image sequence for a successful docking, where the robot did not need to back up, is illustrated. The estimation errors close to the pallet are small which ensures that the robot back up rather than try to dock from a bad final position. The sequence is one of a few initial experiments. The typical orientation repeatability is within $0.6^{\circ}$ (or $10 \mathrm{mrad}$ ). In cross range the standard deviation is within $0.002 \mathrm{~m}$. With better association it will be smaller. In some of the tests one of 
the bevels on the middle block is missed out in the association causing an additional error of $0.008 \mathrm{~m}$.

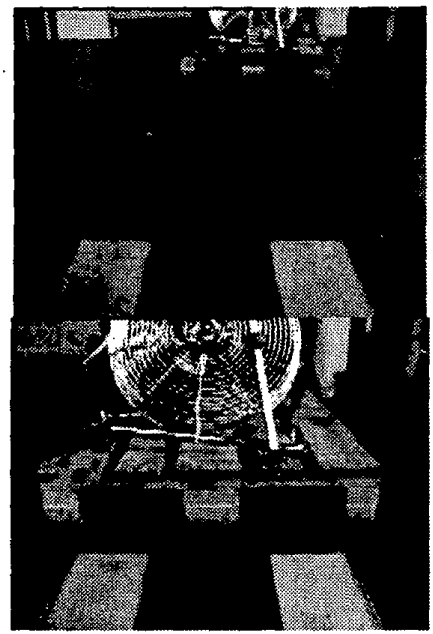

Figure 10 First and last image in a sequence of four from a successful docking. After the final image the following position is straight forwards with the forks into the pallet.

\section{Discussions and future work}

The experiments presented above are preliminary and partially hampered by system integration problems. Considering the acceptable performance during the experiments, introduction of earlier results as in [FLA93] and [NNW97] could only result in smoother trajectories.

In [NNW97], an algorithm towards tolerance controlled gripping was presented. Consider the docking task illustrated in Figure 11. Given an accepted failure probability $P_{f}$, the tolerance box $A$ defined by the tolerances $\left[\begin{array}{lll}\varepsilon_{x} & \varepsilon_{y} & \varepsilon_{\theta}\end{array}\right]$ and the goal position $X_{g}=\left[\begin{array}{lll}x_{g} & y_{g} & \theta_{g}\end{array}\right]$ (defined relative to the object). The task is considered reliable if ;

when the task is finished

$$
\operatorname{Pr}\left\{\left[x_{g}-x_{\text {rabot }} \quad y_{g}-y_{\text {rabor }} \quad \theta_{g}-\theta_{\text {rabot }}\right] \notin A\right\}<P_{f}
$$

Note that the goal-coordinates are defined relative to the object and the uncertainty in this pose varies with several order of magnitude during the approach to the object. In sections 2 and 3, calculations leading to an approximation of the uncertainty in this pose were presented.

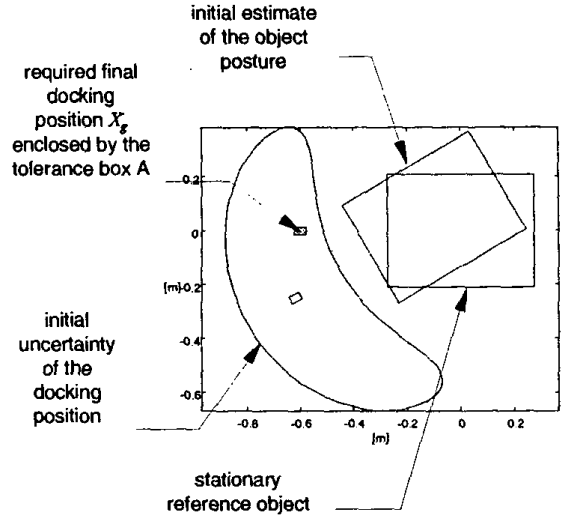

Figure 11 A docking task. To achieve successful docking the final position must be inside the grey rectangle that is the projection' of the tolerance box $A$.

For measurements of a pallet using a sheet-of-light range camera, the uncertainty varies as $r^{2.5}$ in range, $r$ in cross range and $r^{2.5}$ in orientation; (the same applies for the pallet). The proposed control law takes into consideration that the uncertainty has a essentially quadratic relation to the range to the object. Both before and during the motion towards the docking point the failure probability is predicted using a Gaussian mixture model of the final position probability density function. If the final tolerances are not likely to be attained the feedback controller is adjusted. In this way the translational velocity is reduced and more observations of the object are received.

Using the measurement models developed in Section 3, the geometric feedback loop can define a trajectory for the internal robot controller to follow. With the current level of system integration the LQG-based control law from [NNW97] is replaced by postures selected by optimization as in [NN96]. The postures are then forwarded to the robot control. Experiments with this control law are under way.

A conclusion from the present experiments as well as in [NNW97] is that the weak part when using non-contact sensing for feedback in robots is the association problem.

The results on door passage in [FLA93] are similar to the current docking task. Here a range-measuring laser is used instead of the sheet-of-light camera. However, since the orientation in this case is measured relative to the whole wall, the uncertainty does not vary as much. Hence the control law does not have to account for varying

1. The full tolerance box extends into the orientation dimension. 
uncertainty during the approach to the door. The same applies for several other "visual servoing" cases where a stationary camera is used for servoing of a mobile robot or a gripper. Note that this makes the design of a control law simpler, but the final error is not necessarily smaller. The resolution in the sensor in these cases is fixed, and if not small enough to fulfill the tolerances of the task, the system will fail. In the case of a range-camera mounted on the moving robot the sensor moves gradually closer to the target in the finish, thus, allowing smaller tolerances to be met.

\section{Acknowledgment}

The authors thank NUTEK and CENIIT for financial support. Also, the help with hardware by and with the experiments by the participants of THHP01, Calle Lindvall and Niclas Forrestier is greatly appreciated.

\section{References}

[AM79] Anderson, B. D. O. and Moore, J. B. Optimal Filtering, Prentice Hall, 1979

[Bes88] Besl, P.J., "Active, Optical Range Imaging Sensors," Machine Vision and Applications, pp. 127$152,1988$.

[BSF88] Bar-Shalom Y. Fortmann T. E. Tracking and Data Association, Academic Press Vol. 179, 1988

[FAW97] Forsberg, J., Aarenstrup, R., Wernersson, A., "A Construction Robot for Autonomous Plastering of Walls and Ceilings", IAARC ISARC-14, Pittsburgh PA, USA June 1997.

[FLÅ93] Forsberg, J., Larsson, U., Åhman, P. and Wernersson, $\AA$., "The Hough Transform inside the Feedback Loop of a Mobile Robot", IEEE R\& A Soci. Int. Conf. on Robotics \& Automation pp 791798 , Atlanta, GA, USA 1993

[For98] Forsberg, J., Mobile Robot Navigation Using Non-Contact Sensors, Doctoral thesis Luleå University of Technology, November 1998.

[GoS88] Gordon, S. J. and Seering, W. P., "Real-Time Part Position Sensing," IEEE Trans. on Pattern Analysis and Machine Intelligence., Vol. 10, No. 3, pp. 374-386, May 1988.

[Hyp86] Hyyppä, K., "Optical Navigation System using Passive Identical Beacons," Proc. Intelligent Autonomous Systems, Amsterdam, 1986.

[LiC92] Li, Z. and Canny, J. F., Eds. Nonholonomic Motion Planning., Kluwer, 1992.
[Nil97] Nilsson, B., On Robot Control using Range Sensors with Special Reference to Active Uncertainty Reduction, PhD thesis, Dissertation No. 511, Linköping University, Sweden, 1997.

[NN96] B. Nilsson and J. Nygårds "Sensor Motion Planning with Active Uncertainty Reduction: Gripping and Docking Tasks," Proc. IEEE Int. Conf. on Robotics and Automation, Vol. 4 pp. 3026-3033, Minneapolis, April 22-28,1996.

[NNL99] Nilsson, B., Nygårds, J., Larsson, U. and Wernersson, $\AA$., "Control of Flexible Mobile Manipulators: Positioning and Vibration Reduction using an Eye-in-Hand Range Camera," IFAC, Control Engineering Practice 7, pp. 741-751, Elsevier, 1999.

[NNW97] Nilsson, B., Nygårds, J. and Wernersson, $\AA$., "On-Range Sensor Feedback for Mobile Robot Docking Within Prescribed Posture Tolerances," Journal of Robotic Systems Vol. 14 No. 4, pp. $297-$ $312,1997$.

[NW98] Nygårds, J. and Wernersson, Å. "On Covariances for Fusing Laser Rangers and Vision with Sensors Onboard a Moving Robot," Proc. IEEE/RSJ Int. Conf. on Intelligent Robots and Systems IROS'98, Victoria, Oct. 1998.

[Nyg98] Nygårds, J., On Robot Feedback from Range Sensors: Reliable Control by Active Reduction of Uncertainty and Ambiguities, PhD thesis, Dissertation No. 528, Linköping University, Sweden, 1998.

[SpV89] Spong, M. W., and Vidyasagar, M., Robot Dynamics and Control, Wiley, 1989. 